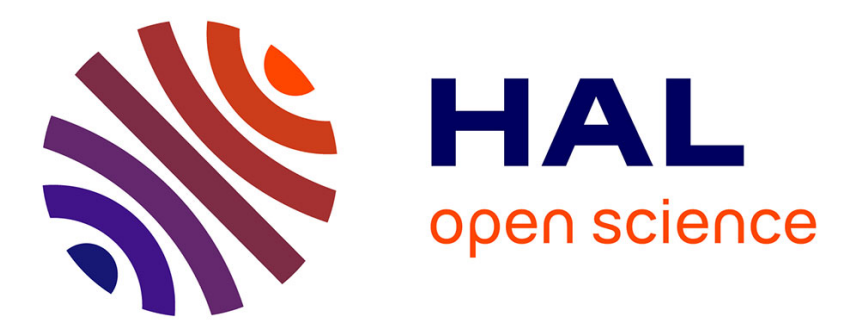

\title{
A novel design of cubic stiffness for a Nonlinear Energy Sink (NES) based on conical spring
}

\author{
Donghai Qiu, Sébastien Seguy, Manuel Paredes
}

\section{To cite this version:}

Donghai Qiu, Sébastien Seguy, Manuel Paredes. A novel design of cubic stiffness for a Nonlinear Energy Sink (NES) based on conical spring. International Joint Conference on Mechanics, Design Engineering \& Advanced Manufacturing,14-26 septembre 2016, Catane, Italie, Sep 2016, Catane, Italy. pp.565-573. hal-02047820

\section{HAL Id: hal-02047820 \\ https://hal.science/hal-02047820}

Submitted on 1 Jul 2021

HAL is a multi-disciplinary open access archive for the deposit and dissemination of scientific research documents, whether they are published or not. The documents may come from teaching and research institutions in France or abroad, or from public or private research centers.
L'archive ouverte pluridisciplinaire HAL, est destinée au dépôt et à la diffusion de documents scientifiques de niveau recherche, publiés ou non, émanant des établissements d'enseignement et de recherche français ou étrangers, des laboratoires publics ou privés. 


\title{
A novel design of cubic stiffness for a Nonlinear Energy Sink (NES) based on conical spring
}

\author{
${\text { Donghai } \text { QIU }^{1 *} \text {, Sébastien SEGUY }}^{1}$ and Manuel PAREDES ${ }^{1}$ \\ ${ }^{1}$ Institut Clément Ader (ICA), Université de Toulouse, CNRS-INSA-ISAE-Mines Albi-UPS, \\ 3 rue Caroline Aigle, 31400 Toulouse, France \\ * Corresponding author. Tel.: +33 (0) 5611711 80; E-mail address: qiu@insa-toulouse.fr
}

\begin{abstract}
Mitigation of unwanted vibration is an important issue in aeronautics and space area. Since the emergence of innovative absorber Nonlinear Energy Sink (NES), more attentions were paid to this promising technique. This absorber is characterized by a secondary mass highly coupled via a nonlinear stiffness to the main structure that needs to be protected. The mastery of the nonlinearity is a key element for obtaining optimum performance for NES. However, it is difficult to implement cubic stiffness without linear part. In this paper, a novel design NES of cubic stiffnes without linear part is presented. For this, the two conical springs are specially sized to provide the polynomial components only with linear and cubic term. To counterbalance the linear term, a concept of negative stiffness mechanism is implemented by two cylindrical compression spring. A small sized NES system is developed. To validate the concept, the load-displacement relation test is performed, and simulation under period excitation and transient loading is studied. Future developments will aim experimental validation and application of the prototype.
\end{abstract}

Keywords: Nonlinear Energy Sink; cubic stiffness; conical spring; negative stiffness mechanism; strongly modulated response

\section{Introduction}

Vibration mitigation devices are required more rigorous with the modern mechanical products designed faster, lighter and more sophisticated. Particularly in the aeronautics and space, it requires the absorber be light, and have a broad spectrum of frequency. For the traditional linear absorber is hard to achieve these requests, thus introducing the nonlinear energy sink (NES) seems to be a good way of dealing these issues. This type of absorber is characterized by a secondary mass highly coupled via a nonlinear stiffness to the main structure that needs to be protected [1]. It is firstly investigated by Gendelman and Vakakis, and it has demonstrated that this system with strongly nonlinear element is able to absorb and dis- 
sipate the energy efficiently from the main structure [2]. With the performance, a wide variety of applications of NES can be used in space and aero-structure, vibrating machinery, building and vehicle suspensions.

The concept of a NES relies on a vanishing linear stiffness, giving rise to an essentially nonlinear restoring force. Having no natural frequency, the NES can thus adapt itself to the frequency of the primary system. A targeted energy transfer (TET) can then occur in an irreversible fashion [3]. Depending on the type of nonlinearity, NES can be categorized as cubic NES, vibro-impact NES, piecewise NES, and rotational NES. As far as the cubic NES, it has been shown that this configuration is most effective at moderate-energy regimes. However, this NES hasn't been applied broadly in engineering practice. One key issue is that, it is difficult to implement cubic stiffness without linear part. In our recent approaches, the essential cubic stiffness was mostly realized by employing the construction of two transverse linear springs with no pretension [4]. However, this type may not be suitable for practical application, due to its large size; in addition, a relatively weak nonlinear stiffness exists at the beginning extension, leads to the cubic term approximated to a linear term.

Another idea which has emerged recently is to use nonlinear spring in translation direction. For example, it has been show in [5] by adopting elastomeric spring with pyramidal shape to provide a nearly cubic restoring force, yet this type's may be limited with the request of large displacement of NES. Therefore, implementing a cubic stiffness element practically is still an important issue to broaden the application of NES.

In this article, a novel NES design leading to the award of a strongly cubic stiffness is presented. The structure is as follows: section 2 is devoted to the theoretical design, including the conception of conical springs and negative stiffness mechanism; in section 3, the assembly of a small sized NES system is developed. In the next section, simulation validation of this NES system under period excitation and transient loading is studied. Finally, concluding remarks and future developments are addressed.

\section{The theoretical design}

\subsection{Conical spring}

Considering the strong nonlinearity and avoid buckling at large deflections, two telescoping conical springs with a constant pitch are adopted. The dynamical behavior of conical spring with a constant pitch can be classified as linear and nonlinear part. In the linear phase, the largest coil is free to deflect as the other coils, so the load-deflection relation is linear and the stiffness can be expressed as:

$$
R=\frac{G d^{4}}{2 n_{a}\left(D_{1}^{2}+D_{2}^{2}\right)\left(D_{1}+D_{2}\right)}
$$


In the nonlinear regime, the first elementary part of the largest coil has reached its maximum physical deflection. It starts to be a non-active element of the spring. During the second regime of compression, the active coils continuously decrease leads to a gradual increase of the spring stiffness. The load-deflection relation is shown as follows:

$$
\Delta(\mathrm{P})=\frac{2 P D_{1}^{4} n_{a}}{G d^{4}\left(D_{2}-D_{1}\right)}\left[\left(1+\left(\frac{D_{2}}{D_{1}}-1\right) \frac{n_{f}}{n_{a}}\right)^{4}-1\right]+\left(L_{a}-L_{s}\right)\left(1-\frac{n_{f}}{n_{a}}\right)
$$

Where the detailed description of Eq. (1) and Eq. (2) can refer to [6].

To benefit the nonlinear performance of conical spring, the symmetrical connecting type of spring is proposed as shown in Fig.1.

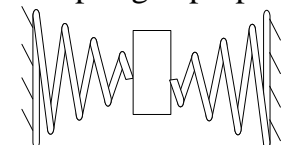

Fig.1 Symmetrical connecting type
Fig.2 Pre-compressing at transition point

However, this configuration has the disadvantage that stiffness curve is piecewise. The dynamical behavior of spring is still classified as the linear and nonlinear part. To skip the linear phase, a method of pre-compressing at transition point is adopted, as shown in Fig.2. By changing the initial original point, the behaviors of two conical springs in Fig. 3 can respectively belong to linear and nonlinear regimes simultaneously.

Combining the two spring's curves, the composed stiffness curve in Fig.4 is obtained and it is obviously observed that the new curve is smooth and no longer piecewise.

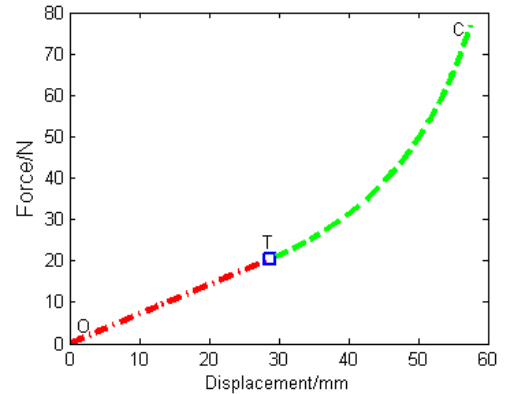

Fig.3 Conical spring characteristic

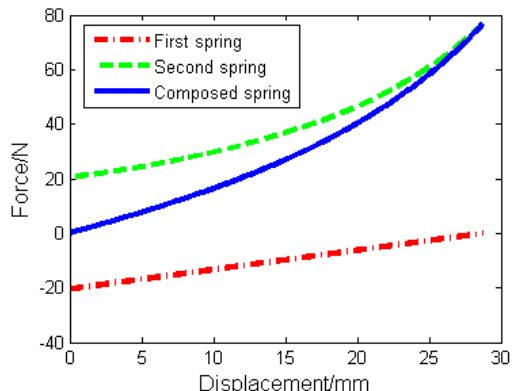

Fig.4 Pre-compressed characteristics

To analyse the internal polynomial components, the method of polynomial fitting is adopted and the new load-deflection relation is expressed as follows:

$$
P=a_{1} x+a_{2} x^{2}+a_{3} x^{3}
$$

In this polynomial, the linear term $a_{1} x$ is hardly to be eliminated owing to the superposition of linear and nonlinear part, while the square term $a_{2} x^{2}$ is possible to make its value small. 
Fig. 5 is the characteristic of the composed components. As the coefficient of square term is large, the curve of cubic and linear term can't fit the original curve well. In addition, it's hard to decrease linear term meanwhile keep the cubic term at the fixed range. So next step, the work of optimization will be introduced.

\subsection{Optimization design}

To describe the stiffness curve, the objective function of conical spring is set as piecewise as follows:

$$
F=\left\{\begin{array}{l}
k_{0} x \quad\left(x \leq s_{t}\right) \\
a_{3}\left(x-s_{t}\right)^{3}+a_{2}\left(x-s_{t}\right)^{2}+a_{1}\left(x-s_{t}\right)+p_{t} \quad\left(x>s_{t}\right)
\end{array}\right.
$$

Where $k_{0}$ is the rate of the linear phase, $p_{t}$ and $s_{t}$ correspond respectively to the force and the displacement of the transition point.

The optimization model consists of the following part: (a) Objective: setting absolute values of square coefficient $\left|a_{2}\right|$ as minimum; (b) Variable: mean diameter of smallest coil $D_{1}$ and mean diameter of largest coil $D_{2}$ (These two values are the main factors to determine the nonlinearity), the coil diameter $d$, number of active coils $n_{a}$, free length $L_{0}$; (c) Constraints: the cubic coefficient $a_{3}$, the deflection of transition point $s_{t}$, the linear stiffness $k_{0}$; (d) Optimization method: Multi-island Genetic Algorithm (MIGA). This algorithm has the advantage of solving the huge domain space problems.

After optimization, a new polynomial component is obtained and presented in Fig.6. It can be observed that the curve of cubic a nd linear term is close to the original one, by means that the contribution of square term is small that can be almost neglected.

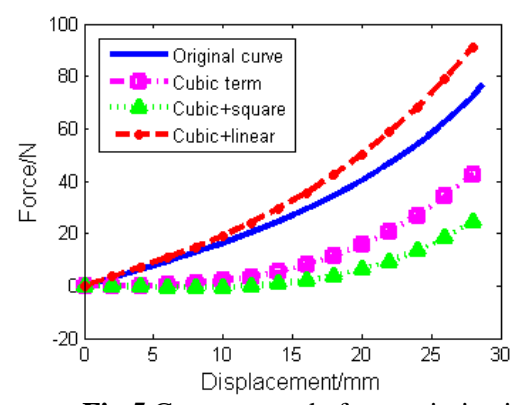

Fig.5 Components before optimization

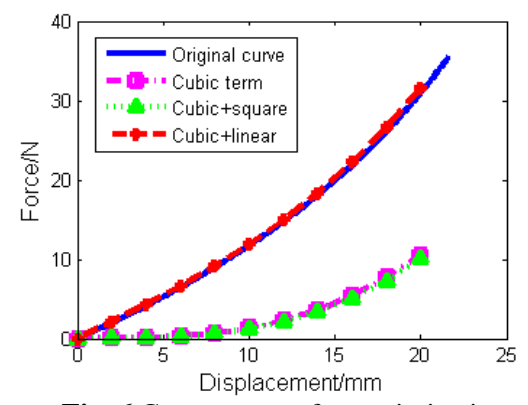

Fig. 6 Components after optimization

\subsection{Negative stiffness}

In order to counteract the linear term of conical and have a pure cubic forcedisplacement, adding a new term which has the negative stiffness in translation direction seems be a way forward. For this, a negative stiffness mechanism is implemented from two cylindrical compression springs, and the structure is shown in Fig.7. 


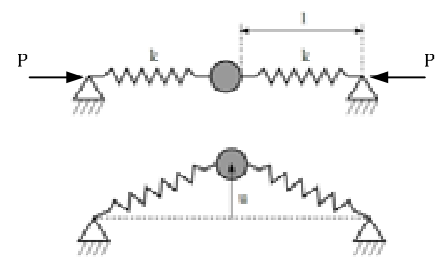

Fig.7 Negative stiffness mechanism

Where $l$ is the free length of linear spring, $P$ is the pre-compressing force, $l_{p}$ is the pre-compressing length, $u$ is the displacement of NES.

After pre-compressing spring in the length of $l_{p}$, the force-displacement relationship based on Taylor expansion is given as:

$$
f=2 \frac{P}{l} u-\frac{k l+P}{l^{3}} \cdot u^{3}
$$

Superposing the force with the one of conical spring in the translational direction, the composed force can be expressed as:

$$
P_{m}=\left(a_{1}-2 k \frac{l_{p}}{l}\right) \cdot x+\left(a_{3}+k \frac{l_{p}+l}{l^{3}}\right) \cdot x^{3}
$$

According to the equation, if we set $a_{1}=2 k l_{p} / l$, the linear component could be canceled out by the negative stiffness mechanism. The equation will be left with the pure cubic term, and its coefficient will increase a little larger.

\section{Assembly of NES system}

Based on the proposed methods, a small sized NES system providing strongly nonlinear stiffness is designed, and the assembly drawing is presented in Fig.8, the component parts are spherical plain bearing, linear guide, conical spring, linear spring and NES mass, of which $x$ and $y$ correspond respectively to the displacement of primary system and NES mass. It is important to highlight that the spring distance to NES mass is adjustable so to reach the suitable force shape.

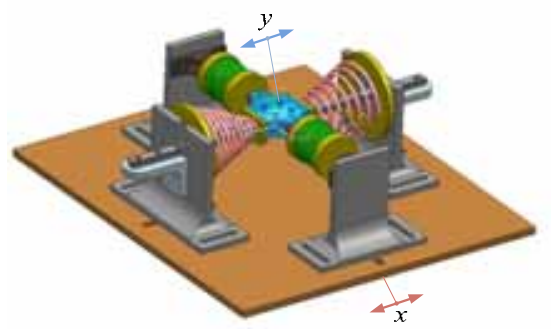

Fig.8 Assembly of NES system 


\subsection{Spring test}

To obtain the performance of conical spring, identification study is performed. The conical spring manufactured is presented in Fig.9, and the test equipment is shown in Fig.10. Here we test five piece conical springs, and the results are obtained in Fig.11. As expected, the linear part of experimental curves fits the theoretical curve perfectly, and the nonlinear part is close to theoretical one.

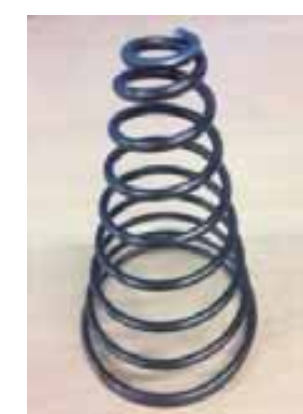

Fig.9 Telescoping conical spring

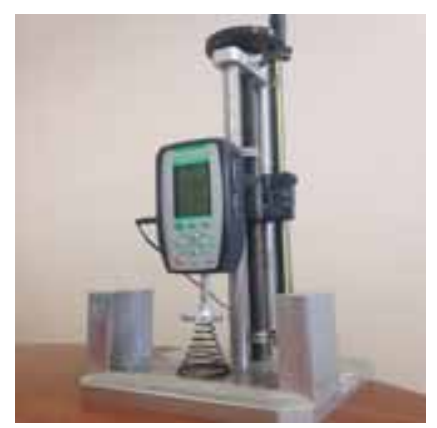

Fig.10 Compression spring test

To make sure the conical spring work in compression state, the maximum displacement of NES is limited at the deflection of transition point. The corresponded characteristic curve is presented in Fig.12. It can be shown that the composed force of conical spring and negative stiffness mechanism corresponds well to the theoretical one, by means this novel conception can be extended to design the strongly cubic NES without linear part.

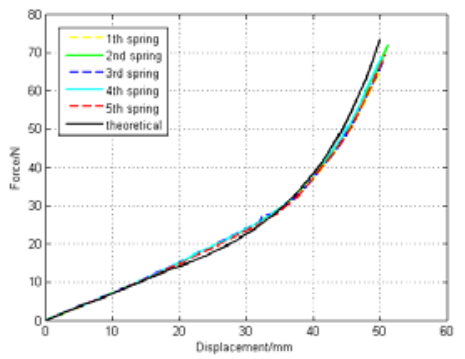

Fig.11 Stiffness curve of manufactured spring

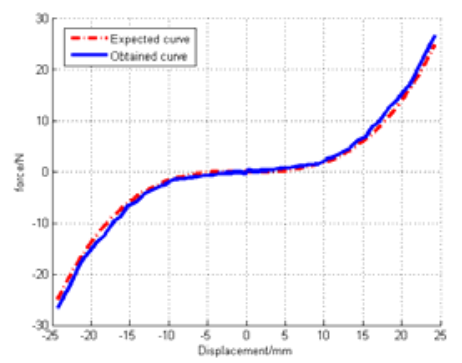

Fig.12 Stiffness curve of NES system

\subsection{NES mass calculation}

The mass of NES $m_{N E S}$ consists of main mass, spherical plain bearing, linear spring support base, and conical spring support base. As the mass of the NES is very small, the inertia of the springs is no longer negligible and has to be considered. In a rough approximation, considering the spring as a beam and neglecting axial inertia, the kinetic energy of the NES mass and linear spring is written as follows: 


$$
T_{N E S}=\int_{0}^{l_{0}} \rho_{s}\left(\frac{x}{l_{0}} \dot{y}\right)^{2} d x+\frac{1}{2} m_{N E S} \dot{y}^{2}
$$

Where $\rho_{s}=m_{\text {linear }} / l_{0}$ is the mass density of the spring. Thus the effective mass of single linear spring can be expressed as $m_{\text {linear }}=m_{l} / 3$, the detailed process can refer to [4] and [7].

For the single conical spring, the effective mass is written as follows.

$$
m_{\text {conical }}=2 m_{c} \frac{\frac{1}{10}\left(1-\beta^{10}\right)-\frac{1}{3} \beta^{4}\left(1-\beta^{6}\right)+\frac{1}{2} \beta^{8}\left(1-\beta^{2}\right)}{\left(1-\beta^{4}\right)^{2}\left(1-\beta^{2}\right)}, \quad \beta=\frac{R_{2}}{R_{1}}
$$

Based on this, the total mass of NES can be depicted as:

$$
m_{2}=m_{N E S}+2 m_{\text {linear }}+2 m_{\text {conical }}
$$

\section{Simulation validations}

\subsection{Dynamical model}

Based on the previous test, the parameters of NES system is obtained in Tab.1. To validate this concept, an analytical study of a harmonically excited linear oscillator (LO) strongly coupled to a NES is presented.

Tab.1 Parameters of NES

\begin{tabular}{clll} 
Tab.1 Parameters of NES & & \\
\hline$m_{1}$ & $9 \mathrm{~kg}$ & $k_{2}$ & $2.33 \times 10^{6} \mathrm{~N} / \mathrm{m}^{3}$ \\
$m_{2}$ & $0.09 \mathrm{~kg}$ & $C_{1}$ & $4 \mathrm{Ns} / \mathrm{m}$ \\
$k_{1}$ & $3 \times 10^{4} \mathrm{~N} / \mathrm{m}$ & $C_{2}$ & $0.4 \mathrm{Ns} / \mathrm{m}$ \\
\hline
\end{tabular}

The governing equations of motion of this system are given by:

$$
\begin{aligned}
& m_{1} \frac{d^{2} x}{d t^{2}}+c_{1} \frac{d x}{d t}+k_{1} x+c_{2}\left(\frac{d x}{d t}-\frac{d y}{d t}\right)+k_{2}(x-y)^{3}=k_{1} x_{e}+c_{1} \frac{d x_{e}}{d t} \\
& m_{2} \frac{d^{2} y}{d t^{2}}+c_{2}\left(\frac{d y}{d t}-\frac{d x}{d t}\right)+k_{2}(y-x)^{3}=0
\end{aligned}
$$

Where the imposed harmonic displacement $x_{e}=G \cos \tilde{\Omega} t$.

\subsection{Periodic loading}

Based on the Eq. (10), setting the excitation amplitude as $0.1 \mathrm{~mm}$, the numerical responses of NES and LO is obtained in Fig.13. A quasi-periodic regime with a slow evolution of the amplitudes of both oscillators is observed. For LO, the amplitude increases and decreases repeatedly in a regular fashion. For the amplitude of NES, it can be classified into two levels: a small one corresponding to the growth of LO amplitude, a large one when LO amplitude decreases. This alternating regime of strongly modulated response (SMR) proves the jump 
phenomenon of SIM [8]. The instaneous percentage energy carried by NES and LO is presented in Fig.14. It can be oberved there exists a phase resonance capture, the energy is entirely transferred to the NES which localizes quickly almost 100 percent of the energy of the system.
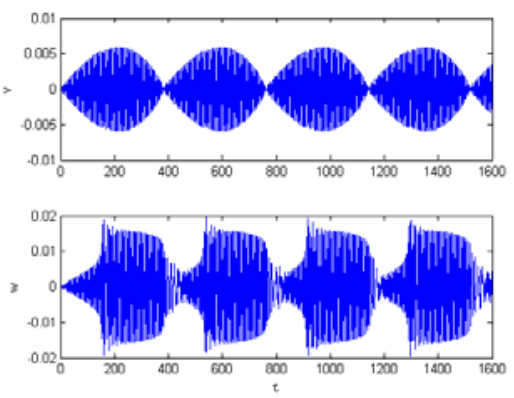

Fig. 13 SMR with $\mathrm{G}=0.1 \mathrm{~mm}$

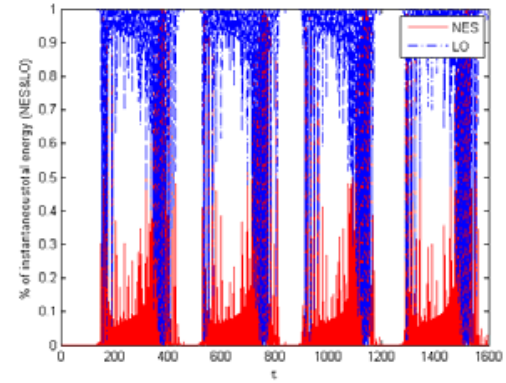

Fig.14 Instaneous energy carried by NES and LO

\subsection{Transient loading}

By introducing the excitation $G=0$ and the initial condition $x=5 \mathrm{~mm}$ to Eq. (10), the numerical response is obtained in Fig. 15. It can be observed, without NES, the vibration extinction of LO will follow a natural exponential decrease; while with NES, it will follow a quasi-linear decrease, much faster than the exponential one, during which the NES will vibrate with a large amplitude until the energy in LO has been almost completely cancelled. In Fig. 16, the evolution of the energy and the percentage of the energy present in NES and LO is showed. We can find that the energy is transferred irreversible from LO to NES, of which almost 68 percent of the energy is dissipated by NES.
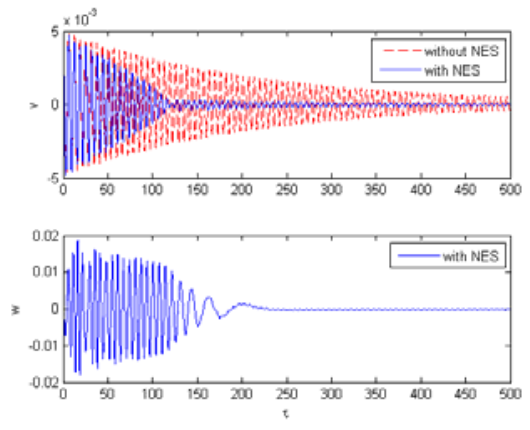

Fig. 15 Transient response

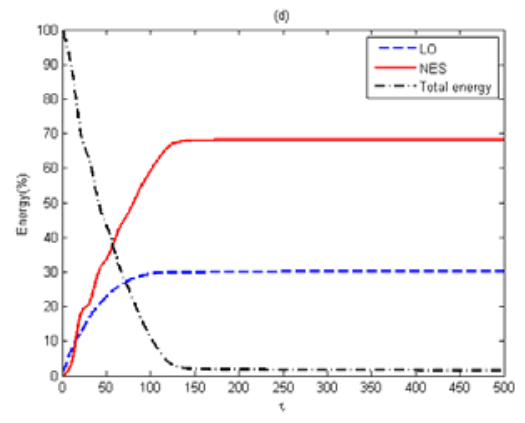

Fig.16 Evolution of the energy in NES and LO

\section{Conclusion}

In this paper, a novel design NES of cubic stiffnes without linear part is presented. For this, two conical springs are specially sized to provide the polyno- 
mial components only with linear and cubic term. To counterbalance the linear term, the concept of negative stiffness mechanism is proposed by two cylindrical compression spring. A small sized NES system providing strongly nonlinear stiffness is developed, of which the distance of spring is adjustable so to reach the suitable force shape. To validate the concept, the load-displacement relation test is performed, and the simulation of a harmonically excited linear oscillator coupled to this type of NES is studied. The results show that this structure can output the pure cubic stiffness as expected; at the specified period excitation amplitude, this system could passively transfer the unwanted disturbance energy with the response of strongly modulated response (SMR); at transient loading, this type can dissipate targeted energy irreversibly with the speed of vibration extinction of LO following a quasi-linear decrease. Future developments will aim experimental validation, and the application of passive vibration control in spatially flexible structures.

\section{References}

1. Gendelman O, Manevitch L I, Vakakis A F, et al. Energy pumping in nonlinear mechanical oscillators: Part I-Dynamics of the underlying Hamiltonian systems[J]. Journal of Applied Mechanics, 2001,68(1): 34-41.

2. Kerschen G, Kowtko J J, McFarland D M, et al. Theoretical and experimental study of multimodal targeted energy transfer in a system of coupled oscillators[J]. Nonlinear Dynamics, 2007, 47(1-3): 285-309.

3. Lee Y S, Vakakis A F, Bergman L A, et al. Passive non-linear targeted energy transfer and its applications to vibration absorption: a review[J]. Proceedings of the Institution of Mechanical Engineers, Part K: Journal of Multi-body Dynamics, 2008,222(2): 77-134.

4. Gourc E, Michon G, Seguy S, et al. Experimental investigation and design optimization of targeted energy transfer under periodic forcing[J]. Journal of Vibration and Acoustics, 2014,136(2): 021021.

5. Luo J, Wierschem N E, Hubbard S A, et al. Large-scale experimental evaluation and numerical simulation of a system of nonlinear energy sinks for seismic mitigation[J]. Engineering Structures, 2014, 77: 34-48.

6. Rodriguez E, Paredes M, Sartor M. Analytical behavior law for a constant pitch conical compression spring[J]. Journal of Mechanical Design, 2006, 128(6):1352-1356.

7. Yamamoto Y. Spring's Effective Mass in Spring Mass System Free Vibration [J]. Journal of Sound and Vibration, 1999, 3(220): 564-570.

8. Gendelman O V, Gourdon E, Lamarque C H. Quasiperiodic energy pumping in coupled oscillators under periodic forcing[J]. Journal of Sound and Vibration, 2006,294(4): 651-662. 